Revista de Derecho

de la Pontificia Universidad Católica de Valparaíso

XXXV (Valparaíso, Chile, 2010, 2 Semestre)

[pp. 79-105]

\title{
El TELETRABAJO COMO UNA ALTERNATIVA PARA PROMOVER Y FACILITAR LA CONCILIACIÓN DE RESPONSABILIDADES LABORALES Y FAMILIARES
}

["Telework as an Alternative to Encourage and Make Work and Family

Responsibilities Harmonization easier"]

Eduardo CaAmaño Rojo*

Pontificia Universidad Católica de Valparaíso

\begin{abstract}
RESUMEN
En un contexto como el actual, en que se cuestiona el modelo de "hombre proveedor y mujer cuidadora" que subyace al Código del Trabajo por su incidencia negativa en la materialización de la igualdad de oportunidades entre trabajadoras y trabajadores, el desarrollo y utilización de modalidades atípicas de contratación puede abrir nuevas opciones para reequilibrar los roles de género en materia laboral. En efecto, formas de contratación como el teletrabajo pueden ofrecer soluciones que no se traduzcan en la postergación de la realización profesional para las mujeres que optan por la maternidad, como asimismo, generar oportunidades nuevas para que los hombres satisfagan su creciente interés por asumir más activamente la paternidad, desligándose de su tradicional
\end{abstract}

\begin{abstract}
In our current context, where "the male breadwinner and the woman caretaker" model that underlies the Labor Code due to its negative incidence in the materialization of equality of opportunity between female and male workers is being questioned, the development and use of atypical modalities of hiring can create new options to balance gender roles in labor matters. In fact, job-hiring modes, such as telework, may offer solutions that do not mean that women have to postpone their professional fulfillment if they choose maternity and create new opportunities for men to satisfy their growing interest to undertake their paternity in a more active way, disassociating themselves from their traditional role of mere breadwinners. This study attempts to evidence
\end{abstract}

* Doctor en Derecho por la Universidad de Colonia, Alemania, profesor de Derecho del Trabajo en la Escuela de Derecho de la Pontificia Universidad Católica de Valparaíso. Dirección postal: Avda. Brasil 2950, Valparaíso, Chile. Dirección electrónica: eduardo.caamano@ucv.cl 
papel de meros proveedores del sustento. El presente estudio busca poner de manifiesto la forma en que el teletrabajo puede contribuir a generar oportunidades para la conciliación de trabajo y familia, con el fin de contribuir a la necesaria concreción de la equidad de género en el país.

Palabras Clave: Teletrabajo - Familia y trabajo. the way in which telework can contribute to create opportunities for the harmonization of work and family in order to contribute to the necessary definiteness of gender equity in the country.

KeYwords: Telework - Family and work.

\section{Planteamiento del problema de la conciliación de Vida}

\section{LABORAL Y FAMILIAR DESDE LA PERSPECTIVA}

\section{DEL DERECHO DEL TRABAJO}

En los últimos años se han producido importantes cambios en el ámbito empresarial y laboral, entre los que destaca la creciente incorporación de la mujer al mercado de trabajo, lo que ha generado una serie de repercusiones tanto en la ordenación del trabajo al interior de las empresas, como en la vida familiar, por lo que trabajadores y trabajadoras han tenido que comenzar a replantearse de manera cada vez más intensa su rol en el empleo y en la familia. Esto último es especialmente significativo, si se considera que la normalidad de las relaciones laborales fue construida a partir de la realidad de los hombres trabajadores. Por consiguiente, el desafío contemporáneo desde una mirada social, cultural, jurídica y económica es reorientar esa normalidad a partir del reconocimiento de la igualdad de hombres y mujeres, potenciando también medidas que posibiliten armonizar las implicancias que para el trabajo tiene la dimensión personal y familiar de trabajadores y trabajadoras.

Este fenómeno social que ha comenzado a tomar cada vez más fuerza en la última década, ha tenido una fuerte incidencia en la regulación jurídica del trabajo por cuenta ajena y en las instituciones tradicionales del Derecho del Trabajo, las que han tenido que comenzar a readaptarse a esta nuevo escenario. En efecto, es evidente el Derecho del Trabajo se construyó a partir de la necesidad de tutela de un conglomerado de trabajadores (hombres, mujeres y niños) afectados por la cuestión social de fines del siglo XIX y principios del siglo XX, por lo que su normativa es reflejo de esa realidad histórica y social. Ahora bien, en ese contexto, la respuesta del Derecho a la problemática social generó un curioso efecto, toda vez que la participación laboral femenina comenzó a disminuir notoriamente, dado que la mujer a cambio de su protección como trabajadora y madre debió asumir en solitario 
el cuidado de los hijos y de la familia, entregándose a los hombres la función de proveer el sustento familiar ${ }^{1}$.

En la actualidad, las transformaciones económicas y sociales del trabajo han puesto en evidencia que los sistemas jurídicos clásicos inspirados en el impuesto paradigma "del hombre proveedor y la mujer cuidadora" no han estado preparados para responder a las interrogantes que hoy representan los problemas derivados de la incidencia de la discriminación por causa de sexo y la demanda por conciliar responsabilidades laborales y familiares, todo lo cual ha llevado inexorablemente a tener que comenzar a rediseñar la legislación laboral. Así, entonces, ocurre que el cuestionamiento de los roles de género tradicionales y la imperiosa necesidad de un número creciente de personas por armonizar su vida personal y familiar con el trabajo, en especial en los casos de familias monoparentales, ha significado que esta problemática haya salido de la esfera estrictamente privada para convertirse en un problema público relacionado con el bienestar de la familia.

Por tanto, desde el punto de vista del Derecho, la reformulación de los roles de género frente al trabajo y la familia adquiere aristas de enorme relevancia social, ya que por una parte, dicen relación con el reconocimiento efectivo del derecho de las mujeres a poder desarrollarse en su dimensión laboral (y no sólo familiar o de cuidado) con igualdad de oportunidades que los hombres. A lo anterior, cabe agregar, por otra parte, el interés cada vez más significativo de los padres trabajadores de poder asumir de modo más directo e intenso sus responsabilidades familiares, lo que hoy resulta muy difícil al negarles el ordenamiento jurídico laboral prácticamente todo derecho en este sentido, a pesar de que la legislación civil los reconoce como co-responsables del cuidado de sus hijos ${ }^{2}$. Sin perjuicio de lo anterior, el tema en análisis cobra vigencia, puesto que involucra también los intereses superiores de los niños y niñas ${ }^{3}$, quienes son titulares de derechos fundamentales, entre otros, el de poder recibir el cuidado, atención y cariño de su padre y de su madre ${ }^{4}$, lo

${ }^{1}$ Véase: CAAmaño Rojo, Eduardo, Mujer y trabajo: origen y ocaso del modelo del padre proveedor y la madre cuidadora, en Revista de Derecho de la Pontificia Universidad Católica de Valparaiso, 34 (semestre I de 2010), p. 179.

${ }^{2}$ En efecto, el artículo 224 CC. prescribe que: "Toca de consuno a los padres, o al padre o madre sobreviviente, el cuidado personal de la crianza y educación de sus hijos"

${ }^{3}$ De conformidad con lo previsto por el artículo 222 inciso $2^{\circ}$ CC.: "La preocupación fundamental de los padres es el interés superior del hijo, para lo cual procurarán su mayor realización espiritual y material posible, y lo guiarán en el ejercicio de los derechos esenciales que emanan de la naturaleza humana de modo conforme a la evolución de sus facultades".

${ }^{4}$ Así se desprende del artículo 18 inciso $1^{\circ}$ de la Convención de los derechos del niño, el cual dispone que: "Los Estados Partes pondrán el máximo empeño en garantizar el reconocimiento del principio de que ambos padres tienen obligaciones comunes en lo que 
que es dificultoso de materializar, si sus progenitores no tienen posibilidades concretas de conciliar trabajo y vida familiar.

Ante esta realidad, pensamos que Chile se encuentra hoy en una encrucijada histórica y en el momento preciso para replantear el viejo pacto de producción y reproducción a partir del reconocimiento efectivo la igualdad de hombres y mujeres frente al trabajo y la familia. Esta necesidad de cambio es evidente, si se considera que en la actualidad nuestro país presenta deplorables niveles de participación laboral femenina que lo ubican en el nivel más bajo dentro de América Latina 5 . Lo anterior, implica a su vez un notable desaprovechamiento de la fuerza laboral femenina, cada día más capacitada y explica, en parte, la paulatina disminución de la tasa de natalidad, dado que para muchas mujeres la opción de trabajar o de ser madre se transforma en una alternativa irreconciliable. Urge, por tanto, asumir decisiones estratégicas que favorezcan el trabajo decente con corresponsabilidad social y un crecimiento con equidad de género.

Además, desde la perspectiva del Derecho del Trabajo chileno, subsisten deficiencias estructurales que no han podido solucionarse a través de reformas legales más bien parciales, las que se han limitado a reconocer la igualdad formal de hombres y mujeres, sin atacar las causas profundas que explican la subsistencia de la discriminación femenina ${ }^{6}$. Se requiere, por lo tanto, una reforma sistémica que posibilite la reconstrucción del sistema tutelar de los trabajadores asentado en la premisa irreductible de la igualdad real entre hombres y mujeres. Refuerza esta necesidad, el compromiso que el país aún mantiene pendiente en orden a adecuar nuestro ordenamiento jurídico laboral al contenido esencial de la Convención sobre eliminación de toda forma de discriminación en contra de la mujer y al Convenio $\mathrm{N}^{\circ}$ 156 de la Organización Internacional del Trabajo sobre los trabajadores con responsabilidades familiares.

Por lo anterior, entonces, el presente estudio pretende contribuir al replanteamiento de las instituciones tradicionales del Derecho del Trabajo con el fin de abrir nuevas oportunidades para superar las brechas de inequidad

respecta a la crianza y el desarrollo del niño. Incumbirá a los padres o, en su caso, a los representantes legales la responsabilidad primordial de la crianza y el desarrollo del niño. Su preocupación fundamental será el interés superior del niño".

${ }^{5}$ En detalle: Dirección del Trabajo, Encuesta Laboral 2008: Inequidades y brechas de género en el empleo, disponible en: http://www.dt.gob.cl/documentacion/1612/w3article-97629.html.

${ }^{6}$ Véase: CAAmaño Rojo, Eduardo, Los efectos de la protección a la maternidad para la concreción de la igualdad de trato entre hombres y mujeres en el trabajo, en Revista de Derecho de la Pontificia Universidad Católica de Valparaiso, 33 (semestre II de 2009), p. 175 s. 
que separan a trabajadoras y trabajadores. Un objetivo de esta naturaleza se puede alcanzar en la medida que el respeto de los derechos fundamentales de la persona sea un eje rector de esta rama del Derecho ${ }^{7}$, en particular, en lo referido a la igualdad de trato de hombres y mujeres, para lo cual la reformulación de las tradicionales modalidades de contratación laboral, o bien, la utilización de nuevas formas de contratación surgidas al alero del desarrollo tecnológico del último tiempo, posibiliten avanzar en el establecimiento de un nuevo equilibrio en los roles de género. Esto último es precisamente uno de los aportes que puede ofrecer el teletrabajo, pues más allá de sus ventajas como instrumento de flexibilidad laboral horaria o funcional, esta variante del contrato de trabajo presenta oportunidades de conciliación de trabajo y vida familiar que se pueden convertir en una alternativa eficaz para trabajadores y trabajadoras, cuya situación laboral les permita prestar servicios a distancia, avanzando así en la superación de un modelo normativo construido aún en torno al reconocimiento de la madre trabajadora como única responsable del cuidado de la familia.

\section{ANTECEDENTES, CONCEPTO Y CARACTERÍSTICAS DEL TELETRABAJO}

En el contexto general de las transformaciones experimentadas por el trabajo subordinado a fines del siglo XX y principios del actual ocupa un lugar preponderante el impacto de las nuevas tecnologías, tanto en lo que respecta a su inserción en los procesos productivos, como en lo que se refiere a su creciente utilización en el marco de la prestación de servicios personales ${ }^{8}$.

La revolución generada por el desarrollo de la informática y las demás tecnologías de la información, más su rápida introducción a los procesos productivos ha contribuido a la diversificación de la producción en períodos cortos de tiempo ${ }^{9}$. Por ello, de acuerdo a lo planteado por Raso ${ }^{10}$, las nuevas tecnologías provocan una verdadera revolución en el campo de las relaciones laborales, lo que se puede resumir en tres aspectos: a nivel individual permiten

${ }^{7}$ En lo que respecta a la incidencia y proyección de los derechos fundamentales en materia laboral véase: Ugarte Cataldo, José Luis, El nuevo Derecho del Trabajo (Santiago, Editorial LexisNexis, 2007), p. 115 s.

${ }^{8}$ Sobre la incidencia de las nuevas tecnologías en el contrato de trabajo: GAMONAL Contreras, Sergio, Trabajo y Derecho (Santiago, Editorial Legal Publishing, 2010), p. $35 \mathrm{~s}$.

${ }^{9}$ Véase: Jáuregui Atondo, Ramón - Moltó García, Juan Ignacio - GonZÁLEZ DE LENA, Francisco, Un futuro para el trabajo en la nueva sociedad laboral (Valencia, Editorial Tirant lo Blanch, 2004), p. 61 s.

${ }^{10}$ Raso Delgue, Juan, Las transformaciones del trabajo, p. 7. Este documento está disponible en: www.rau.edu.uy.universidad/instderechodeltrabajo/raso.pdf. 
a las personas multiplicar su capacidad de acción, lo que determinará la sustitución de grandes contingentes de mano de obra por sofisticadas máquinas y la promoción de un reducido número de trabajadores con la suficiente formación y capacitación para operarlas; a nivel colectivo, se produce una fragmentación del modelo de relaciones laborales, por un lado, pues ya no será fácil organizar trabajadores con intereses y condiciones económicas muy diferentes y, por otro lado, existirá una gran movilidad de las empresas en un mercado que favorecerá a aquellas que optaron por la reconversión industrial y condenará a la quiebra a las que no se adecuaron a los cambios; finalmente, las nuevas tecnologías y la reconversión promueven nuevas formas de gestión del trabajo, que procurarán fundamentalmente una mayor productividad y competitividad. Es decir, con el desarrollo de las herramientas informáticas y su incorporación a las industrias y a las empresas de servicios se ha posibilitado el paso de la producción en serie y estandarizada a la producción diversificada, a pedido y, por tanto, altamente flexible y variable, con lo que es posible responder a los requerimientos cambiantes de los consumidores.

Por otra parte, cabe hacer presente que el desarrollo e incorporación de nuevas tecnologías a los procesos productivos ha generado profundos cambios en las habilidades laborales requeridas a los trabajadores, por lo que el trabajo parcelado y repetitivo propio del modelo de organización fordista-taylorista va perdiendo peso estratégico ${ }^{11}$, ya que las competencias laborales que demandan las empresas son cada vez más integrales, al suponer una combinación de destrezas manuales e intelectuales, porque en la medida que la rígida estructura vertical va dando paso a una visión más horizontal de la empresa, el trabajo en grupo, el liderazgo, la creatividad y otras cualidades laborales comienzan a ser altamente valoradas por la moderna administración de recursos humanos ${ }^{12}$. En este contexto, estas nuevas calificaciones otorgan, en principio, una mayor autonomía en la planificación y ejecución de las tareas para los trabajadores y, en ese sentido, del manejo de sus propios tiempos, si bien, no exento de riesgos, en cuanto pueden volverse menos claros los límites tradicionales de separación entre el tiempo de trabajo y aquel destinado al descanso o a la vida familiar ${ }^{13}$.

${ }^{11}$ Sobre la materia: CAAmaño Rojo, Eduardo, Las transformaciones del trabajo, la crisis de la relación laboral normal y el desarrollo del empleo atípico, en Revista de Derecho de la Universidad Austral de Chile, 18 (julio de 2005), p. 25 s.

${ }^{12}$ En detalle: Dessler, Gary, Administración de Personal ( $8^{a}$ edición, México, Editorial Pearson, 2001), p. 13 s.

${ }^{13}$ Véase: Díaz,Ximena; Todaro, Rosalba,Riesgoseinseguridadesdelasnuevasformas deusoflexibledeltiempodetrabajo, en SerieEn Foco,35 (2004), disponible en: http://www. expansiva.cl/publicaciones/en_foco/detalle.tpl?iddocumento $=06102004132859$ 
A su vez, cabe tener en consideración que en la actualidad se incrementa la posibilidad de realizar las actividades laborales en diversos espacios físicos y fuera del "establecimiento" de la empresa, lo que es producto de la fragmentación de los procesos productivos y de los servicios y, en parte, de los límites más difusos que posibilita la tecnología entre tiempo de trabajo, de ocio o de no trabajo ${ }^{14}$. De igual forma, se debe tener en cuenta que la proliferación de las formas atípicas de empleo está relacionada con la creciente flexibilización de la organización interna de la empresa y con el proceso de descentralización productiva.

Es esta perspectiva, según lo plantea el Consejo Económico y Social de España ${ }^{15}$ a partir de la cual se presenta la teoría de la "empresa flexible" de Atkinson ${ }^{16}$, que se correspondería con lo que se podría identificar como "empleo del futuro", ofreciendo una visión premonitoria de hacia dónde camina el modelo de mercado de trabajo actual. Pues bien, según esta teoría la organización de la empresa se plantea como un sistema de círculos concéntricos. El núcleo central estaría compuesto por un grupo de trabajadores, si no fijos, sí dotados de unas garantías fuertes de seguridad en el empleo -la relación laboral normal mantendría respecto de estos trabajadores su papel de modelo de ordenación del empleo subordinado- y que estarían encargados de desempeñar las funciones clave, permanentes o de más responsabilidad en la empresa, a los que se aplicaría un alto grado de flexibilidad funcional ${ }^{17}$. Alrededor de este núcleo giraría un círculo de trabajadores periféricos, gracias a los cuales la empresa obtendría la suficiente flexibilidad numérica ${ }^{18}$ y cuyo

${ }^{14}$ Sobre la materia: Rieuelme, Verónica, El tiempo de trabajo, en Temas Laborales, 11 (Dirección del Trabajo), documento disponible en: www.dt.gob.cl.

${ }^{15}$ Consejo Económico y Social, El trabajo a tiempo parcial, Informe № 4 (Sesión ordinaria del pleno de fecha 18 de septiembre de 1996, Madrid, 1996), p. 5.

${ }^{16}$ Atkinson, J. - Meager, N., New Forms OfWork Organisation, IMS, Report No 89 (Breighton, 1986), citado por Consejo Económico y Social, El trabajo a tiempo parcial, cit. (n. 15), p. 5.

${ }^{17} \mathrm{La}$ flexibilidad funcional dice relación con la posibilidad de adaptar las funciones convenidas con los trabajadores en virtud de sus respectivos contratos de trabajo, frente a un cambio de las circunstancias en las que se desarrolla el proceso productivo. De esta manera, se exige que el trabajador sea capaz de asumir tareas diversas ante necesidades también diversas, con lo cual el nuevo perfil laboral se aleja del arquetipo de trabajador propio del modelo fordista-taylorista, caracterizado por su bajo nivel de formación y por asumir tareas repetitivas y uniformes. Expresiones concretas de la flexibilidad funcional en Chile son, por ejemplo, la denominada "polifuncionalidad" (artículo 10 No 3 CdT.) y el "ius variandi" (artículo 12 inciso $1^{\circ} \mathrm{CdT}$ ).

${ }^{18}$ Esta clase de flexibilidad, denominada también flexibilidad externa, corresponde a la posibilidad de realizar ajustes en la mano de obra disponible para la empresa frente a necesidades económicas determinadas, ya sea aumentándola vía contratación (flexibilidad de entrada), o bien, disminuyéndola a través de despidos (flexibilidad de salida). 
volumen oscilaría en función de las fluctuaciones de la demanda. La relación de estos trabajadores con la empresa se basaría en distintas modalidades de contratación, fundamentalmente de duración determinada y entre las que el trabajo a tiempo parcial ocuparía un lugar preeminente, conviviendo con otros como los contratos de obra y servicio de corta duración, el trabajo de fin de semana o nocturno y algunas modalidades subvencionadas para el fomento del empleo. El tercer círculo de este modelo sería el denominado "empleo a distancia", caracterizado por el mayor grado de desvinculación con la empresa, en el que se incluirían relaciones como el teletrabajo y la subcontratación.

Queda clara, entonces, la importancia manifiesta de las formas atípicas de ocupación en el mercado laboral del siglo XXI, por cuanto éstas representan en gran medida el cauce natural a través del cual se están canalizando los cambios más significativos del trabajo en la actualidad, pues le proporcionan a las empresas y a los mismos trabajadores las posibilidades de superar las rigideces o deficiencias de la relación laboral normal para hacer frente a los desafíos que conlleva la superación del modelo fordista en la organización empresarial.

Por consiguiente, no se puede desconocer que las nuevas tecnologías y, en particular las denominadas tecnologías de la información y la comunicación (TIC's) han proyectado también su incidencia en la organización de las relaciones laborales, reflejada en la figura tradicional o normal del contrato de trabajo, con lo cual comienzan a surgir nuevas modalidades de contratación laboral o formas atípicas. Estas formas atípicas de contratación laboral se diferencian del modelo típico, pues falta en ellas algunos de sus elementos (v. gr. la jornada a tiempo completo o la duración indefinida del contrato), o bien, porque modifican algunos de los elementos fundantes del empleo fordista, como es la localización de la prestación de los servicios en el lugar físico de la empresa.

Este es el caso particular del teletrabajo, el cual se va configurando como una modalidad atípica de trabajo, y fue definido por primera vez en la década de 1970 por Jack Nilles ${ }^{19}$ como "cualquier forma de sustitución de desplazamientos relacionados con la actividad laboral por tecnologías de la información”. Más recientemente, Thibault ${ }^{20}$ define al teletrabajo como

En detalle: Ugarte Cataldo, José Luis, Derecho del Trabajo, flexibilidad laboral y análisis económico del Derecho (Santiago, Editorial LexisNexis, 2004), p. 36 s.

${ }^{19}$ Nilles, Jack, The Telecommunications-Transportation Tradeoff. Options for Tomorrow and Today (California, 1973), citado por: ThiBAult ARANDA, Javier, El teletrabajo. Análisis jurídico laboral, Colección Estudios No 88, 2a edición (Consejo Económico y Social, Madrid, 2001), p. 24.

${ }^{20}$ Thibault Aranda, Javier, El teletrabajo, cit. (n. 19), p. 32. 
"una forma de organización y/o de ejecución del trabajo realizado en gran parte o principalmente a distancia, y mediante el uso intensivo de técnicas informáticas y/o de telecomunicación".

Por su parte, en Chile, el teletrabajo ha sido definido como: "una forma flexible de organización del trabajo que consiste en el desempeño de éste fuera del espacio habitual de trabajo, durante una parte importante de su horario laboral, pudiendo realizarse a tiempo parcial o completo"21. El autor citado agrega a continuación como una particularidad del teletrabajo el que éste engloba una amplia gama de actividades y requiere el uso frecuente de tecnologías de información y comunicación para el contacto entre el trabajador y la empresa, pudiendo ser realizado por cualquier persona independiente del género, edad y condición física.

De la definición anterior y siguiendo lo planteado por $\operatorname{Ugarte}^{22}$ y por Thibaul $t^{23}$, se puede establecer que el teletrabajo constaría de tres elementos distintivos:

a) Elemento geográfico o locativo: esta particularidad supone que el teletrabajo es ante todo un tipo de trabajo a distancia -de allí su similitud con el trabajo a domicilio ${ }^{24}$ - por lo que para calificar una determinada prestación de servicios como una modalidad de este tipo, se requiere que los servicios sean prestados fuera del centro físico de funcionamiento de la empresa. Con todo, se debe entender que no se trata necesariamente de una prestación de servicios desde el domicilio del trabajador, sino que puede implicar servicios prestados desde cualquier localidad o lugar ubicado fuera de la empresa o centro de trabajo dirigido y controlado por el empleador.

b) Elemento tecnológico o cualitativo: además del trabajo a distancia, el teletrabajo requiere como uno de sus elementos definitorios la utilización intensiva de una determinada tecnología que permita precisamente el desarrollo de una tarea productiva fuera del centro de funcionamiento de la

${ }^{21}$ SAlazar Concha, Cristian, El teletrabajo como un aporte a la inserción laboral de personas con discapacidad en Chile: una gran carretera virtual por recorrer, en Revista Ciencia \& Trabajo, 25 (julio-septiembre de 2007), p. 89, disponible en www.cienciaytrabajo.cl.

${ }^{22}$ Ugarte Cataldo, José Luis, El nuevo Derecho del Trabajo, cit. (n. 7), p. 181183.

${ }^{23}$ Thibault Aranda, Javier, El teletrabajo, cit. (n. 19), p. 29-31.

${ }^{24}$ Un completo estudio sobre el trabajo a domicilio realiza: MonTENEGRo ÁviLA, Natalia, Trabajo a domicilio a la luz del derecho internacional, comparado y chileno (memoria para optar al grado de licenciada en Ciencias Jurídicas, Pontificia Universidad Católica de Valparaíso, Valparaíso, 2009). Véase también: Henríquez, Helia - CÁrdenas, Ana - Selamé, Teresita, Trabajo a domicilio en el siglo XXI. Tres miradas sobre el teletrabajo, en Cuaderno de Investigación, 26 (Santiago, Dirección del Trabajo, 2005). 
empresa. Estas tecnologías son básicamente las informáticas y las de telecomunicaciones (ejemplo: redes computacionales, correo electrónico, teléfono, videoconferencia, etc.) y posibilitan en la práctica que la prestación de los servicios del trabajador se canalice por estos medios hacia el empleador. Es precisamente el uso intensivo de estas tecnologías, lo que permite distinguir el teletrabajo del trabajo a domicilio, pues este último está caracterizado por el intercambio de bienes materiales cuantificables y fungibles entre el empleador, quien los proporciona, y el trabajador, quien le agrega valor a través de su trabajo ${ }^{25}$.

c) Elemento organizativo: Para Thibault ${ }^{26}$, teletrabajar no es sólo trabajar a distancia y utilizando las telecomunicaciones y/o la informática, pues a su juicio teletrabajar es servirse de estos elementos para prestar servicios de un modo diferente. Por consiguiente, no sería teletrabajador todo aquel que emplea las herramientas propias del teletrabajo (por ejemplo un computador portátil), sino sólo aquel que por el hecho de utilizarlas escapa al modelo tradicional de organización del trabajo. Por lo tanto, se debe entender que el teletrabajo es una forma de organización y de realización de trabajo distinta por parte de una persona natural, en un contexto de descentralización de actividades laborativas que antes eran realizadas en la empresa.

En definitiva, con la conjunción de estos elementos el teletrabajo adquiere una identidad propia y, en cuanto modalidad de contratación laboral, interesará para el Derecho del Trabajo aquella que es prestada en condiciones de ajenidad y subordinación, esto es, bajo la dirección y control de la parte empleadora, con lo cual adquiere relevancia la respuesta normativa del ordenamiento jurídico laboral que posibilite la construcción de un sistema diferenciado de protección para responder así a sus particularidades que alejan naturalmente al teletrabajo de una relación laboral normal o típica.

\section{LA REGULACIÓN DEL TELETRABAJO en el Código del Trabajo}

Como ya se ha señalado, el teletrabajo es una modalidad atípica de contratación laboral que se ha ido perfilando con una identidad propia, en la medida que el progresivo desarrollo y expansión en el uso de las nuevas tecnologías ha permitido alterar o modificar algunos de los elementos definitorios de una relación laboral normal. Por lo anterior, resulta fácil concluir que no es posible entender su inclusión en la legislación laboral, sino en la medida que esta forma de trabajo alcanza un mayor grado de incidencia práctica y

\footnotetext{
${ }^{25}$ Ugarte Cataldo, José Luis, El nuevo Derecho del Trabajo, cit. (n. 7), p. 183.

${ }^{26}$ Thibault Aranda, Javier, El teletrabajo, cit. (n. 19), p. 31.
} 
cuando se hace patente la necesidad de intervenir normativamente, con el fin de proteger los derechos de los trabajadores, armonizándolos con el legítimo interés de los empresarios de adecuar los sistemas de trabajo a la nueva realidad económica tan fuertemente tensionada por el paulatino proceso de globalización ${ }^{27}$.

En el caso chileno, el teletrabajo es reconocido por primera vez a través de la dictación de la Ley No 19.759 de 2001 que lo incorpora como un caso de excepción a la duración máxima de la jornada ordinaria de 45 horas semanales consagrada en el artículo 22 inciso $1^{\circ}$ del Código del Trabajo (= CdT.). De esta manera, el legislador buscó regularizar "el teletrabajo, esto es, aquel que se desarrolla por trabajadores contratados para prestar sus servicios fuera del lugar de funcionamiento de la empresa, mediante la utilización de medios tecnológicos, como pueden ser los informáticos o de telecomunicaciones. Al efecto, se deroga el inciso penúltimo del artículo $8 \mathrm{CdT}$., armonizándose la aplicación de dicho Código con los restantes trabajadores que laboren en un lugar distinto de la empresa, aunque no sea mediante elementos de tecnología y comunicación avanzados" 28 .

El artículo 22 inciso $4^{\circ} \mathrm{CdT}$., en su nueva redacción luego de la reforma legal referida, prescribe que: "Asimismo, quedan excluidos de la limitación de jornada, los trabajadores contratados para que presten sus servicios preferentemente fuera del lugar o sitio de funcionamiento de la empresa, mediante la utilización de medios informáticos o de telecomunicaciones" ${ }^{29}$.

${ }^{27}$ Esta necesidad se confirma con lo expresado en el "Mensaje Presidencial” con el cual se dio inicio a la tramitación de la que luego sería la Ley No 19759. En el acápite I.5, el mensaje establece lo siguiente: "[...] conscientes de las transformaciones en el mercado de trabajo, y principalmente en la estructura del empleo y de la organización del trabajo, se ha estimado necesario incorporar a la legislación del trabajo, diversas fórmulas contractuales de promoción del empleo. Dichas fórmulas buscan constituir un adecuado instrumento de la politica social a través de normas proactivas para la contratación que, sin entorpecer el desarrollo productivo de las empresas, resguarden debidamente al trabajador, contribuyan de otra parte a combatir la precarización del trabajo y su consecuente exclusión social. Se busca, pues, una regulación que establezca normas claras, que incentiven la contratación, pero que otorguen efectiva protección social a quienes contribuyen a generar los bienes y servicios. Por eso, hemos estimado necesario incluir en el proyecto normas que den cuenta de las nuevas tendencias en materia de contratación y de formas promocionales de empleo, asi como la introducción de mecanismos que permitan la adaptabilidad laboral'. Mensaje No 136-343 de fecha 16 de noviembre de 2000.

${ }^{28}$ Acápite II.4.b del Mensaje No 136-343 de fecha 16 de noviembre de 2000.

${ }^{29}$ Cabe tener en consideración que a propósito del sueldo base obligatorio, el artículo 42 letra a) CdT establece que “[...] se presumirá que el trabajador está afecto a cumplimiento de jornada cuando debiere registrar por cualquier medio y en cualquier momento del dia el ingreso o egreso a sus labores, o bien cuando el empleador efectuare descuentos por atrasos en que incurriere el trabajador. Asimismo, se presumirá que el trabajador está 
Según Walker ${ }^{30}$, lo anterior significa que, habiendo subordinación, todos los trabajos que se realicen fuera del lugar de funcionamiento físico de la empresa por medios tecnológicos de carácter informático o de telecomunicaciones deben ser considerados como contratos individuales de trabajo.

En concreto, la subordinación que presupondría el trabajo dependiente estaría sustentada, en el caso del teletrabajo, tanto en la ajenidad de la definición de los contenidos del trabajo a realizar y las características del producto o servicio, así como de su resultado (el producto o servicio), su localización en el mercado y la definición de su precio. Esta ajenidad daría origen a una relación de dependencia económica del trabajador respecto del empleador o dador del trabajo manifestada principalmente por la ejecución de un trabajo en forma continua y estable, el establecimiento de un horario de trabajo, el cual podría también estar determinado por la fecha de entrega del trabajo, y por la percepción de una remuneración como resultado del trabajo efectuado. Asimismo, la dependencia derivada del sometimiento del trabajador a las órdenes y las directrices del empresario se manifestaría en el teletrabajo a través de elementos tales como: la modalidad de inserción del trabajo en el sistema informático y telemático de la empresa (formas de enlace con la empresa, características del programa que dirige la prestación del servicio), la propiedad de la tecnología (si es propietario o no del hardware o software que utiliza) y/o la facultad de determinación de los programas específicos que se utilizarán, así como el nivel de conexión funcional y económica existente respecto del ciclo productivo de la empresa ${ }^{31}$.

Ahora bien, no cabe duda que la opción utilizada por el CdT. para ocuparse del teletrabajo es bastante curiosa, pues lejos de darle una especificidad normativa o de incluir algunas disposiciones que permitan resolver las interrogantes que no son susceptibles de responderse con las reglas generales establecidas para el contrato de trabajo, pareciera, luego de una

afecto a la jornada ordinaria, cuando el empleador, por intermedio de un superior jerárquico, ejerciere una supervisión o control funcional y directo sobre la forma y oportunidad en que se desarrollen las labores, entendiéndose que no existe tal funcionalidad cuando el trabajador sólo entrega resultados de sus gestiones y se reporta esporádicamente, especialmente en el caso de desarrollar sus labores en Regiones diferentes de la del domicilio del empleador". Estas presunciones tienen únicamente por objeto reconocer a los trabajadores el derecho a un sueldo base no inferior al ingreso mínimo mensual, pero son relevantes, toda vez que contribuyen a precisar el alcance de las excepciones a la duración máxima de la jornada semanal y a reducir ciertos riesgos de precarización para los trabajadores como es, en este caso, en lo referido a sus remuneraciones.

${ }^{30}$ Walker Errázuriz, Francisco, Derecho de las relaciones laborales (Santiago, Editorial Universitaria, 2003), p. 278.

${ }^{31}$ Henrí ueze, Helia - Cárdenas, Ana - Selamé, Teresita, Trabajo a domicilio en el siglo XXI. Tres miradas sobre el teletrabajo, cit. (n. 24), p. 37-38. 
primera lectura, que termina precarizando aún más la situación laboral de los teletrabajadores ${ }^{32}$, ya que al no hacerles aplicable el límite de la duración máxima de la jornada de trabajo, los excluye también del control de asistencia o de la posibilidad de pactar jornada extraordinaria ${ }^{33}$. Sin perjuicio de ello, salta a la vista que el régimen legal general sobre el contrato de trabajo resulta insuficiente e ineficiente para responder a las necesidades particulares que podrían presentar los teletrabajadores. Este es el caso, por ejemplo, del alcance que tendría respecto del teletrabajo el deber general de protección del artículo $184 \mathrm{CdT}$. o el ejercicio del derecho a la negociación colectiva. A su vez, llama la atención que se asimile a los teletrabajadores con aquellos dependientes que prestan servicios sin control directo o sin supervigilancia de su empleador, dado que las particularidades del teletrabajo derivadas del uso de las TIC's precisamente posibilita un control de la actividad, lo que puede llegar a ser bastante preciso y, en concreto, del tiempo de trabajo por parte del empleador ${ }^{34}$.

Con todo, como lo manifiesta Ugarte ${ }^{35}$, el curioso método utilizado por el legislador nacional viene a dotar de una suerte de régimen jurídico específico al teletrabajo, zanjando así la extensa discusión doctrinal acerca de la difusa frontera del teletrabajo entre el empleo subordinado y el empleo autónomo. Por lo anterior, compartimos su opinión en el sentido de que en Chile, el teletrabajo es sin mayor obstáculo una forma de ocupación subordinada, quedando, en consecuencia, bajo el alero del Derecho del Trabajo. Se trataría de una suerte de presunción legal de laboralidad especial, complementaria a la del artículo 8 inciso $1^{\circ} \mathrm{CdT}$. por el sólo efecto de prestar servicios a

\footnotetext{
${ }^{32}$ En igual sentido: Gamonal Contreras, Sergio, Trabajo y Derecho, cit. (n. 8), p. 43.

${ }^{33}$ La Dirección del Trabajo mediante el dictamen No 215/13 de fecha 18 de febrero de 2002 ha establecido que debe dejarse constancia en el respectivo contrato de trabajo de que el trabajador se encuentra en alguna de las situaciones de excepción de la duración de la jornada de trabajo previstas por el artículo $22 \mathrm{CdT}$.

${ }^{34}$ En efecto, "[...] la tecnología informática cambia la forma de controlar el trabajo, permitiendo que la vigilancia se haga a distancia y disminuyendo la importancia de la comunicación cara a cara en un mismo lugar de trabajo. El empleo a domicilio se ha expandido precisamente en sectores productivos y actividades económicas que se pueden organizar y controlar a distancia, a través de la comunicación moderna de base informática o telefónica, como es el trabajo intelectual, el de buena parte de las ocupaciones de profesionales y el de muchas ocupaciones administrativas de oficina. Se suele decir que el control a distancia puede ser más preciso, acucioso e inmediato que el que se ejerce en el mismo recinto (como el trabajo en línea)". En este sentido: HenRíQUEZ, Helia - Cárdenas, Ana - Selamé, Teresita, Trabajo a domicilio en el siglo XXI. Tres miradas sobre el teletrabajo, cit. (n. 24), p. 18.

${ }^{35}$ Ugarte Cataldo, José Luis, El nuevo Derecho del Trabajo, cit. (n. 7), p. 193194.
} 
través de medios informáticos o de telecomunicaciones, sin perjuicio que se pueda probar que los servicios se prestan con el carácter de autónomos, en la medida que ello corresponda a la realidad de ejecución de los mismos, en concordancia con los principios que inspiran y dan forma a esta rama del Derecho.

No obstante lo dicho, es evidente que la incorporación genérica de los teletrabajadores al modelo normativo establecido en favor de los trabajadores subordinados no permite promover esta forma de ocupación entre empresarios y trabajadores, ni satisfacer los requerimientos particulares de protección ante situaciones nuevas que no fueron pensadas o concebidas para el esquema de la relación laboral normal. Esta constatación explica, por tanto, el interés del Gobierno por perfeccionar la regulación de los trabajos a distancia y del teletrabajo, para lo cual envió recientemente un proyecto de ley que desea hacerse cargo de estas deficiencias, según se analizará en el acápite siguiente.

\section{COMENTARIOS AL PROYECTO DE LEY}

\section{SOBRE EL CONTRATO ESPECIAL DE TRABAJO A DISTANCIA}

El proyecto de ley sobre el contrato de trabajo a distancia ${ }^{36}$ pretende perfeccionar la legislación con miras a formalizar las actuales relaciones laborales a distancia, entre las que se ubica el teletrabajo, de tal forma de evitar la precariedad y superar las actuales deficiencias normativas. Estos objetivos se inspiran también en la necesidad de promover esta forma de empleo, especialmente para trabajadores y trabajadoras que no pueden trabajar en un esquema de empleo típico o normal, contribuyendo así a superar el limitado desarrollo y aplicación práctica del trabajo a distancia, en general, y del teletrabajo en particular. En efecto, el escaso nivel de utilización de las formas de trabajo a distancia queda evidente a la luz de la encuesta CASEN 2009, la que da cuenta de que cerca del 90\% de los trabajadores prestan servicios en dependencias de la empresa y que sólo un 3\% trabaja desde su domicilio o teletrabaja ${ }^{37}$.

Por otra parte, cabe destacar que el proyecto tiene también como una de sus principales consideraciones "el proveer de un mecanismo que facilite, especialmente, la incorporación de la mujer, los jóvenes, los adultos mayores y las personas con discapacidad al mundo laboral, como una forma de promover

\footnotetext{
${ }^{36}$ Boletín No 7199-13, de fecha 8 de septiembre de 2010 de la Cámara de Diputados. El proyecto de ley es de fecha 20 de agosto de 2010 y versa sobre el contrato especial de trabajo a distancia.

${ }^{37}$ La Encuesta CASEN 2009 está disponible en: http://www.mideplan.cl/ca$\operatorname{sen} 2009 /$.
} 
nuevas fuentes de trabajo. Es decir, la nueva legislación permitirá conciliar de mejor manera el desarrollo de labores remuneradas con la vida familiar, junto a otras actividades culturales, educacionales, deportivas o recreativas, las que contribuyen decisivamente a mejorar la calidad de vida de laspersonas" ${ }^{38}$. Este objetivo resulta de especial interés en la línea del presente estudio, ya que busca ofrecer alternativas específicas a los trabajadores y trabajadoras con responsabilidades familiares, para lo cual se contempla una interesante propuesta de adecuar los contratos de trabajo a las necesidades particulares que puedan tener hombres o mujeres durante ciertas etapas de sus trayectorias laborales, según se analizará en el apartado siguiente.

Sin perjuicio de lo expuesto, se debe destacar que el proyecto de ley no pretende derogar el actual inciso $4^{\circ}$ del artículo $22 \mathrm{CdT}$., sino que lo mantiene e incorpora una nueva categoría de teletrabajo (también exento de límite de la jornada semanal $)^{39}$, que sería precisamente el que quedaría cubierto con la nueva normativa que se propone, lo cual no está exento de críticas, toda vez que amplía las diferencias ya existentes entre el teletrabajo y el régimen general del contrato de trabajo. Por cierto, con este criterio propuesto por el Ejecutivo, el teletrabajo que no sea catalogado como trabajo a distancia seguirá entregado a la nebulosa e incierta normativa actual, mientras que el teletrabajo que se califique como trabajo a distancia quedará en un plano relativamente mejor, pues el proyecto reconoce algunos derechos que no quedan claros conforme al régimen general del contrato de trabajo ${ }^{40}$. Así, entonces, llama la atención que en su formulación normativa esta propuesta resulte abiertamente contradictoria con el fin de ampliar la tutela de los teletrabajadores y de dar respuesta a las deficiencias que presenta la actual legislación, por lo que es un punto que debería ser objeto de revisión meticulosa y de una adecuada discusión durante la tramitación de esta iniciativa legal.

Ahora bien, el texto del propuesto artículo 86 bis CdT. define en su inciso $1^{\circ}$ al trabajo a distancia, señalando que: "Las normas del presente Capitulo se aplicarán a todos aquellos trabajadores que presten servicios bajo dependencia

${ }^{38}$ Mensaje No 224-358 de 20 de agosto de 2010 con el que se inicia el proyecto de ley sobre el contrato especial de trabajo a distancia, p. 6 .

${ }^{39}$ En efecto, el texto del proyectado artículo 86 bis D dispone que: "Los trabajadores cuyos contratos se rijan por el presente Capitulo, estarán excluidos de la limitación de jornada prevista en el inciso primero del artículo 22 de este Código".

${ }^{40}$ Esto es así, ya que el proyectado artículo 86 bis inciso 3 le reconoce a los teletrabajadores a amparados por este régimen especial los mismos derechos individuales y colectivos de los trabajadores tutelados por el CdT. De igual manera, el artículo 86 bis B establece un contenido mínimo especial para el contrato de trabajo, como a su vez, por destacar sólo algunas de las novedades, se les reconoce en el artículo 86 bis $\mathrm{G}$ un sistema propio de descansos acorde con la naturaleza de los servicios prestados a distancia, todo lo cual no regiría para otras categorías de teletrabajo. 
y subordinación, desde el hogar o un lugar libremente elegido por ellos. Los servicios prestados bajo esta modalidad, también incluyen aquellos que se ejecuten mediante la utilización de medios informáticos o de telecomunicaciones". De lo anterior, se desprende que el elemento identificatorio del trabajo a distancia viene dado por la prestación de servicios subordinados desde el hogar o un lugar libremente determinado por el trabajador. En el caso que, además, los servicios se ejecuten mediante la utilización de medios informáticos o de telecomunicaciones, se estará frente a un tipo de teletrabajo "a distancia" que quedaría sujeto a esta normativa especial. Reafirma esta consideración el texto del inciso $3^{\circ}$ de la norma citada, conforme al cual: "No se aplicarán las normas del presente Capitulo cuando los servicios se presten en lugares designados o habilitados por el empleador, aun cuando se encuentren ubicados fuera de las dependencias de la empresa".

Esta opción del Ejecutivo por diferenciar el trabajo a distancia, estableciendo marcos regulatorios distintos según quien determine el lugar en que deban de prestarse los servicios nos parece muy imprecisa y riesgosa, pues la consecuencia es que en la gran mayoría de los casos será el empleador quien decida donde deban prestarse los servicios, con lo cual quedarán los trabajadores y trabajadoras entregados a la actual falta de certeza sobre el contenido y alcance de sus derechos laborales. Asimismo, la vaguedad de este criterio se ve confirmada con el texto del inciso $4^{\circ}$ del artículo 86 bis, el cual reconoce genéricamente a los trabajadores que prestan servicios a distancia los mismos derechos individuales y colectivos que contiene el CdT., en la medida que no sean incompatibles o contradictorios. Una disposición de esta naturaleza no contribuye a reforzar el reconocimiento y protección de los derechos laborales, pues si bien podría aplicarse sin mayores dificultades para materias tales como el feriado anual o la protección a la maternidad, no resuelve otras interrogantes ${ }^{41}$ que se plantean, como es el caso, por ejemplo, del derecho a la sindicación y a la negociación colectiva, con lo cual puede

${ }^{41}$ Así ocurre, por ejemplo, en materia de seguridad laboral, pues el texto del proyecto se remite a un reglamento que debería dictarse por el Ministerio del Trabajo, sin establecer los criterios, pautas o principios que deberían servir de base a ese texto reglamentario. En efecto, el artículo 86 bis inciso $5^{\circ}$ propuesto por el proyecto establece que: "Las condiciones de higiene y seguridad a que deben sujetarse los trabajadores regidos por este Capitulo, serán reguladas mediante un decreto supremo dictado al efecto por el Ministerio del Trabajo y Previsión Social y suscrito además por el Ministro de Salud. El empleador sólo estará obligado a comunicar por escrito al trabajador dichas condiciones. Durante la vigencia de la relación laboral, corresponderá unicamente al trabajador cumplir las condiciones de higiene y seguridad, en conformidad a las normas que establezca el referido decreto". 
terminar ahondando la ya desmejorada situación en que se encuentra el reconocimiento y ejercicio del derecho fundamental a la libertad sindical ${ }^{42}$.

En definitiva, una mirada general del proyecto de ley permite concluir que los avances en el perfeccionamiento de la situación jurídica laboral que se propone para los trabajadores a distancia y para los teletrabajadores son insuficientes, con lo cual se mantienen muchos de los actuales grados de incerteza y desprotección que tornan poco atractiva a estas modalidades de contratación para las personas que se proyectan como sus principales interesados (v. gr. trabajadores y trabajadoras con responsabilidades familiares). Además, resulta lamentable que no se considere a la negociación colectiva como un medio para dar respuesta particular a las necesidades de los actores sociales en relación con el teletrabajo y los empleos a distancia, como a su vez, que las normas propuestas no se articulen más directamente con la necesaria readecuación que reclama el actual sistema de protección a la maternidad ${ }^{43}$, en concordancia con los planteamientos efectuados por la Comisión Asesora Presidencial "Mujer, Trabajo y Maternidad" ${ }^{4}$.

Conforme a lo expuesto, cabe señalar que si bien el teletrabajo puede representar un modelo no ideal de trabajo para encauzar parte significativa de la trayectoria laboral de una persona, puede llegar a ser -bien utilizado $\mathrm{y}$ con los debidos resguardos a los derechos laborales- un instrumento que contribuya a incrementar la flexibilidad en el uso de los tiempos de trabajo, a fortalecer la autonomía de trabajadoras y trabajadores respecto del tiempo que desean destinar a actividades laborales, a posibilitar la incorporación de nuevas personas al mercado de trabajo, a potenciar el trabajo más cualificado en base a su resultado más que a los tiempos dedicados a su realización, como a su vez, a reconstruir el viejo paradigma de hombre proveedor y mujer cuidadora en base a la equidad de género. Es de esperar, por tanto, que el proyecto de ley, a pesar de sus deficiencias, sea una oportunidad para reflexionar acerca del sentido actual y del futuro de la legislación laboral, posibilitando una mirada renovadora e integradora de las diversas modalidades de contratación laboral.

En último término, como se establecerá en el acápite siguiente, es posible

\footnotetext{
${ }^{42}$ En detalle: Caamaño Rojo, Eduardo - Ugarte Cataldo, José Luis, Negociación colectiva y libertad sindical: un enfoque critico (Santiago, Editorial Legal Publishing, 2008).

${ }^{43}$ Véase: CaAmaño Rojo, Eduardo, Los efectos de la protección a la maternidad para la concreción de la igualdad de trato de hombres y mujeres en el trabajo, en Revista de Derecho de la Pontificia Universidad Católica de Valparaiso, 33 (semestre II de 2009), p. $175 \mathrm{~s}$.

${ }^{44}$ Comisión Asesora Presidencial "Mujer, Trabajo y Maternidad", Informe final, documento disponible en www.sernam.cl.
} 
sostener que, en la actualidad, el teletrabajo puede representar de todas maneras un aporte para promover un nuevo equilibrio entre las responsabilidades laborales y familiares de hombres y mujeres. Para estos efectos, se establecerán algunas propuestas que posibiliten potenciar sus ventajas, minimizando los inconvenientes que presenta el ordenamiento jurídico laboral vigente.

\section{EL TELETRABAJO Y SU POTENCIALIDAD PARA PROMOVER LA ASUNCIÓN EQUILIBRADA DE RESPONSABILIDADES LABORALES Y FAMILIARES}

En la literatura que se ha ocupado del teletrabajo desde una perspectiva funcional ${ }^{45}$, se destacan diversas ventajas del teletrabajo tanto para las empresas, como para los trabajadores, las que son importantes de considerar para el fin que nos interesa, como se señalará más adelante. Es así, como se valorizan como especiales aportes del teletrabajo para las empresas: $i$ ) los incrementos significativos en los niveles de productividad; $i i)$ la reducción del ausentismo laboral; iii) la reducción de costos fijos (arriendos, consumos, mantención, etc.); $i v$ ) la capacidad para atraer y retener trabajadores; $v$ ) su capacidad de alcanzar mayores grados de flexibilidad funcional y horaria; $v i$ ) descentraliza la organización tradicional de la empresa ${ }^{46}$; y vii) genera nuevos servicios o áreas de negocios ${ }^{47}$.

Por su parte, los trabajadores pueden encontrar también diversas ventajas en esta modalidad de contratación laboral, tales como: $i$ ) mayores niveles de satisfacción laboral; ii) una mayor autonomía en el uso de los tiempos de trabajo; iii) ofrece mayores oportunidades de empleo a personas con movilidad reducida; y $i v$ ) una mejor calidad de vida, al facilitarles la creación de un equilibrio entre vivir y trabajar.

Con todo, se advierte también que el teletrabajo presenta algunas desventajas para los trabajadores, pues suele conducir a un aumento en la jornada de trabajo, lo que genera una curiosa paradoja entre trabajar más y mejorar la calidad de vida. Además, el teletrabajo puede conducir a una disminución notoria de las relaciones interpersonales, a un aislamiento laboral y sindical, a una reducción de la actividad física y, en último término

${ }^{45}$ Seguimos sobre este particular a: Salazar Concha, Christian, El teletrabajo como un aporte a la inserción laboral de personas con discapacidad en Chile: una gran carretera virtual por recorrer, cit. (n. 21), p. 90-92; BARRERo FERnÁnDEZ, Antonio, El teletrabajo (Madrid, Editorial Ágata, 1999), p. $117 \mathrm{s.}$

${ }^{46}$ Mensaje No 224-358 de 20 de agosto de 2010 con el que se inicia el proyecto de ley sobre el contrato especial de trabajo a distancia, p. 3.

${ }^{47}$ Ibíd. 
a situaciones desmotivantes ${ }^{48}$. De allí, entonces, que se sostiene que quien desee teletrabajar debe reunir variadas características, como son: un alto nivel de adaptabilidad, capacidad de organización de sus tiempos de trabajo, conocimientos de las TIC's, disposición a capacitación, tenacidad, disciplina, sentido de riesgo, responsabilidad, laboriosidad, tolerancia a la soledad y espíritu emprendedor ${ }^{49}$.

En lo que toca al presente estudio y en la línea de las propuestas para promover y facilitar la armonización de responsabilidades laborales y familiares entre hombres y mujeres, creemos que el teletrabajo, al igual que el trabajo a tiempo parcial ${ }^{50}$, puede representar una opción viable y efectiva en concordancia con un sistema más renovado e igualitario de protección a los trabajadores familiares. En efecto y, sin desconocer las deficiencias que representa la minimalista regulación del CdT. sobre el teletrabajo, es posible sostener que esta modalidad de contratación laboral puede erigirse como una alternativa que permita a mujeres y hombres con responsabilidades laborales familiares, en ciertas etapas de sus trayectorias laborales, poder asumirlas sin desconectarse de sus empleos.

Asimismo, es perfectamente posible, en base a las particularidades de nuestro heterogéneo mercado laboral, que el teletrabajo se convierta en una opción de conciliación de trabajo y familia para trabajadores más calificados, remunerados en base a resultados o tareas, más que por su presencia física en el lugar de la empresa, y que no quieren perder el contacto con su empleador ni ver disminuidos sus ingresos al preferir dedicar más tiempo al cuidado de sus hijos o hijas. En esta misma línea, se puede señalar también que para muchas empresas puede resultar interesante y altamente conveniente que se minimice el impacto de tener trabajadoras o trabajadores con responsabilidades familiares mucho tiempo fuera de la empresa -por ejemplo, por un postnatal extenso- lo que implica perturbaciones de distinta intensidad en el funcionamiento normal de la empresa o costos y problemas asociados a la contratación y capacitación de reemplazantes.

Es lamentable, en todo caso, que dentro de las propuestas presentadas por la Comisión Asesora Presidencial "Mujer, Trabajo y Maternidad" no se contenga ni siquiera una referencia indirecta al teletrabajo, a pesar de haber

${ }^{48}$ Sobre los riesgos de precarización del teletrabajo: HenríQUeZ, Helia - CÁRDENAs, An -; Selamé, Teresita, Trabajo a domicilio en el siglo XXI. Tres miradas sobre el teletrabajo, cit. (n. 24), p. $11 \mathrm{~s}$.

${ }^{49}$ Barrero Fernández, Antonio, El teletrabajo, cit. (n. 45), p. 122-124.

${ }^{50}$ Véase: CAAmaño Rojo, Eduardo, La promoción del trabajo a tiempo parcial como una vía para profundizar la conciliación de trabajo y vida familiar entre hombres y mujeres, en Revista de Derecho de la Empresa, 19 (Universidad Adolfo Ibáñez - Legis Chile, julio-septiembre 2009), p. 103 s. 
sido mencionado reiteradamente en el debate ciudadano que ha suscitado la necesidad de revisar el actual sistema de protección a la maternidad ${ }^{51}$. En efecto, a diferencia de las múltiples referencias al trabajo a tiempo parcial que contiene el informe final de la comisión, era perfectamente posible que se incluyera el teletrabajo como una opción adicional de uso del propuesto "permiso postnatal parental" 52 , el cual podría haberse asumido en forma de teletrabajo a tiempo completo o parcial. En este último caso, el uso del teletrabajo a tiempo parcial habría podido figurar como una opción atractiva, puesto que el informe hace compatible la disminución de la jornada para efectos del cuidado del menor con el subsidio previsto en el artículo 198 $\mathrm{CdT}^{53}$. En nuestra opinión, esta inexplicable omisión por parte de la Comisión Asesora Presidencial devela aún más la escasa incidencia que tiene el teletrabajo en la práctica -de hecho ni la ENCLA 2008 lo considera dentro la información encuestada- así como la necesidad de potenciar sus múltiples ventajas y promover su uso para quienes efectivamente puede representar una alternativa viable para compatibilizar el trabajo con las responsabilidades familiares.

No obstante lo expuesto precedentemente, dadas las falencias de la normativa general sobre contrato de trabajo para enfrentar las dificultades que el teletrabajo podría traer asociadas en la práctica y, dado que no se vislumbran cambios normativos claros sobre la materia, parece recomendable que se establezcan garantías y limitaciones en favor de los trabajadores en los acuerdos contractuales referidos al teletrabajo, bajo el supuesto que esta modalidad de contratación interesa y beneficia a ambas partes. Estas salvaguardas contractuales para reforzar el carácter voluntario del teletrabajo y para aminorar los riesgos de precarización de las relaciones laborales son perfectamente posibles a la luz de los principios generales de la legislación laboral, así como a partir de la consideración de que el ordenamiento jurídico se limita a establecer derechos mínimos e irrenunciables en el plano individual de las relaciones laborales, los que no obstan a acuerdos modificatorios que reconozcan mejores derechos o condiciones laborales a los consagrados en el CdT. Así lo confirma, por lo demás, el artículo 5 inciso $3^{\circ}$ de este cuerpo legal al prescribir que: "Los contratos individuales y colectivos de trabajo podrán ser modificados, por mutuo consentimiento, en aquellas materias en que las partes hayan podido convenir libremente".

\footnotetext{
${ }^{51}$ En este sentido: Bentancor, Andrea - CaAmaño Rojo, Eduardo, Protección a la maternidad: desafíos y oportunidades, Columna de opinión publicada en el Diario La Segunda, miércoles 21 de julio de 2010.

${ }^{52}$ Comisión Asesora Presidencial "Mujer, Trabajo y Maternidad", Informe final, cit. (n. 44), p. 24 s.

${ }^{53}$ Ibíd., p. 25.
} 
La forma de llevar a cabo esta tarea para minimizar los riesgos de precarización de las condiciones laborales en el caso del teletrabajo y potenciarlo de verdad como una vía para avanzar en la materialización de la igualdad real de mujeres y hombres en lo que toca a la asunción de sus responsabilidades laborales y familiares, será objeto de análisis en el apartado siguiente.

\section{OpCIONES DE USO DEL TELETRABAJO DESDE LA PERSPECTIVA}

DE LA CONCILIACIÓN DE RESPONSABILIDADES LABORALES Y

\section{FAMILIARES}

Con miras a convertir al teletrabajo en una opción viable para el fin que inspira el presente estudio y, en concordancia con los principios y normas que dan forma a la legislación laboral, cabe afirmar que una relación laboral de teletrabajo se podría constituir:

i) A partir de la contratación inicial de un trabajador o trabajadora con responsabilidades familiares como teletrabajador.

ii) A partir de la modificación consensuada de un contrato de trabajo entre el empleador y el trabajador o trabajadora, de acuerdo a lo establecido por el artículo 11 inciso $1^{\circ} \mathrm{CdT}$. y con el sentido del principio de continuidad de la relación laboral ${ }^{54}$. En este caso particular, la modificación del contrato de trabajo debería estar centrada a alterar la cláusula del lugar en que se prestan los servicios, así como la cláusula referida a la naturaleza de los servicios que presta el trabajador con el fin de adecuarlas a las particularidades del teletrabajo.

Una alternativa de este tipo es reconocida expresamente por el proyecto de ley sobre trabajo a distancia en el artículo 86 bis E. En particular, la disposición proyectada establece que: "Aquellos trabajadores quepresten sus servicios en la empresa, cumpliendo jornadas de trabajo en conformidad a las reglas generales, podrán pactar con sus empleadores, temporal o permanentemente, que dichos servicios puedan desarrollarse de acuerdo a la modalidad señalada en el inciso $1^{\circ}$ del artículo 86 bis, dejándose constancia de dicho acuerdo en un anexo del contrato de trabajo. Para estos efectos, las partes podrán establecer alternativas de distribución de dias dentro de la semana, o de horas dentro de uno o más días".

${ }^{54}$ Una de las manifestaciones del principio de la continuidad de la relación laboral es la amplitud para la admisión de transformaciones del contrato de trabajo, atendido su carácter dinámico asociado a su carácter de contrato de tracto sucesivo. En detalle: Plá Rodríguez, Américo, Los principios del Derecho del Trabajo ( $3^{a}$ edición, Buenos Aires, Editorial Depalma, 1998), p. 234 s. Véase también: Gamonal Contreras, Sergio, Fundamentos de Derecho Laboral (Santiago, Editorial LexisNexis, 2008), p. 116-119. 
Consideramos que esta norma propuesta por el Ejecutivo es valiosa, pues valida la posibilidad de adecuar los contratos de trabajo acorde con los cambios en la situación personal o de la empresa que puedan experimentar trabajadores, trabajadoras o empleadores, con lo cual se abren nuevas alternativas para conciliar el trabajo con las responsabilidades familiares durante un período de tiempo en el que los menores u otros dependientes requieran cuidado o atención, sin que ello genere una desconexión con su quehacer laboral o profesional. Opciones de este tipo son también valiosas, porque reconocen la heterogeneidad de los trabajadores y de las empresas que es propia de nuestro tiempo, así como la insuficiencia de las normas generales estructuradas a partir de realidades sociales que comienzan a ser repudiadas, como ocurre con el viejo pacto de producción y reproducción que entrega sólo a las mujeres la responsabilidad por el cuidado de la familia.

Sin perjuicio de lo anterior, en el supuesto de modificación de un contrato de trabajo típico en un teletrabajo se podría considerar un acuerdo especial en lo que toca a la jornada de trabajo, ya sea para convenir una excepción a su duración (conforme al artículo 22 inciso $4^{\circ} \mathrm{CdT}$.), ajustar los mecanismos de control de asistencia y de cumplimiento de contrato, si los hubiera, a las particularidades propias del teletrabajo, o bien, para acordar una reducción de la jornada para ajustarla a los límites propios del trabajo a tiempo parcial. Esto último resulta interesante desde una perspectiva de prestación flexible de los servicios y de renovación en el uso de los tiempos de trabajo, toda vez que se podría modificar un trabajo a tiempo completo para transformarlo en un teletrabajo a tiempo parcial (v.gr. pasar de una jornada ordinaria convencional de 40 horas a una a tiempo parcial de 20 horas, en la que los servicios se presten bajo la modalidad de teletrabajo $)^{55}$.

Asimismo, se podría concebir que un contrato de trabajo a tiempo completo de un trabajador o trabajadora con responsabilidades familiares se modifique de tal forma de cumplir una parte de la jornada de trabajo (por ejemplo, la mitad de ella) en el lugar de la empresa, en los términos convenidos al momento de la contratación, y la otra parte en el domicilio del trabajador o la trabajadora bajo la modalidad de teletrabajo ${ }^{56}$.

Por otro lado, para agregar nuevos elementos a esta multiplicidad de alternativas de modificaciones que resultarían posibles a la luz del actual ordenamiento jurídico laboral, se puede estimar que la modificación de un contrato de trabajo típico para convertirlo en un teletrabajo (de jornada

\footnotetext{
${ }^{55}$ En detalle: CaAmaño Rojo, Eduardo, El Trabajo a tiempo parcial (Santiago, Editorial LexisNexis, 2007).

${ }^{56}$ Véase: CAAmaño Rojo, Eduardo, La actual regulación de la jornada de trabajo y su necesidad de flexibilización, en Revista de Derecho de la Pontificia Universidad Católica de Valparaiso, 28 (Semestre I de 2007), p. 337 s.
} 
completa o parcial) puede hacerse por una duración determinada. En efecto, cabría pensar en una modificación transitoria del contrato de trabajo típico en teletrabajo por un plazo determinado (v. gr. 3 meses después de finalizado el permiso postnatal o durante el tiempo del permiso si hubiera una modificación legal en este sentido $)^{57}$, o bien, sujeta al cumplimiento de una condición (por ejemplo, que la salud del niño o niña ya no requiera el cuidado directo de su madre o padre en el hogar).

Con todo, en los casos de modificaciones transitorias de un contrato de trabajo típico en teletrabajo, habría que considerar ciertas garantías para el evento de producirse el término de la relación laboral en aquellos supuestos en que el trabajador o la trabajadora con responsabilidades familiares no se encontrare cubierto por fuero laboral y en los que la modificación pueda haber ido asociada también a una reducción transitoria de las remuneraciones como consecuencia de la reducción de la jornada de trabajo. Ahora bien, si la modificación transitoria hubiese dado lugar a un teletrabajo a tiempo parcial, la respuesta a este riesgo viene dada por el artículo 40 bis D del CdT., el cual establece una regla especial sobre la forma en que corresponde calcular la indemnización por años de servicio en el trabajo a tiempo parcial ${ }^{58}$.

Por el contrario, si la modificación transitoria de un contrato de trabajo típico en un teletrabajo que contenga una reducción de la jornada ordinaria, (obviando la excepción del artículo 22 inciso $4^{\circ} \mathrm{CdT}$.) - por ejemplo de 45 horas a 35 horas semanales- y de las remuneraciones, podrían presentarse problemas en lo que respecta a los alcances de la última remuneración mensual de conformidad al artículo $172 \mathrm{CdT}$. por haber disminuido la base de cálculo de la indemnización. Por ello, podría pensarse en incluir una cláusula

${ }^{57}$ En la actualidad, el artículo $195 \mathrm{CdT}$ concibe al permiso postnatal como un derecho irrenunciable por su función dentro del sistema normativo de relaciones laborales individuales, por lo que no se podría convenir, por ejemplo, que durante las últimas 6 semanas de las 12 que comprende, la trabajadora se reincorpore a la empresa para continuar trabajando a tiempo parcial o como teletrabajadora. Sin embargo, nada obsta a que después del postnatal se reincorpore a tiempo parcial o como teletrabajadora. Además, en este caso, la protección de la trabajadora se vería reforzada con la garantía adicional del fuero maternal consagrado en el artículo $201 \mathrm{CdT}$.

${ }^{58} \mathrm{El}$ artículo 40 bis D CdT establece que: "Para los efectos del cálculo de la indemnización que pudiere corresponderle al trabajador al momento del término de sus servicios, se entenderá por última remuneración el promedio de las remuneraciones percibidas por el trabajador durante la vigencia de su contrato o de los últimos once años del mismo. Para este fin, cada una de las remuneraciones que abarque el periodo de cálculo deberá ser reajustada por la variación experimentada por el indice de precios al consumidor, entre el mes anterior al pago de la remuneración respectiva y el mes anterior al término del contrato. Con todo, si la indemnización que le correspondiere por aplicación del artículo 163 fuere superior, se le aplicará ésta". 
en el pacto modificatorio del contrato que considere como base de cálculo para las indemnizaciones por término de contrato que pudieran corresponder la remuneración anterior a la reducción de la jornada o el promedio de los últimos 3 meses anteriores a la reducción de la jornada de trabajo, si la remuneración fuera variable, o bien, que aplicara por analogía la disposición del artículo 40 bis D CdT..

Sin perjuicio de lo expuesto, cabe hacer presente que las explicaciones anteriores están construidas a partir del reconocimiento de la ya referida heterogeneidad de nuestro mercado de trabajo y pensando en trabajadoras o trabajadores con una capacidad de negociación que les posibilite llegar a este tipo de acuerdos con su empleador, lo que tenderá a ser directamente proporcional con su nivel de cualificación profesional. Esto es, a mayor nivel de calificación laboral o profesional aumentará también la capacidad de negociación de la parte trabajadora y el interés del empleador por minimizar el impacto empresarial que pueda derivarse del ejercicio de la opción por parte del trabajador o trabajadora de asumir un mayor grado de responsabilidades familiares.

Por todo lo anterior, entonces, y con miras a poner también al alcance de un mayor número de trabajadoras y trabajadores el ejercicio de alternativas que posibiliten y promuevan la armonización de trabajo y vida familiar, creemos que una vía plausible y concordante con las necesidades e intereses de los sujetos involucrados es precisamente la negociación colectiva. En efecto, existe un enorme potencial para que sindicatos y empresas avancen de manera significativa hacia relaciones laborales con mayores grados de equidad de género y con corresponsabilidad social, más allá de las posibles reformas legislativas que puedan tener cabida en esta materia en el corto o mediano plazo. Por consiguiente, creemos que puede y debe ser la negociación colectiva un medio para renovar nuestro actual sistema de relaciones laborales, superando las falencias que la negociación política y sectorial de las leyes laborales no puede ofrecer, contribuyendo así a materializar y/o a profundizar un nuevo pacto de producción y reproducción construido a partir del reconocimiento efectivo de la igualdad entre hombres y mujeres.

\section{CONCLUSIÓN}

Para concluir consideramos indispensable reiterar que el tema de la compatibilización entre vida laboral y familiar y el reforzamiento de la real igualdad de oportunidades entre trabajadoras y trabajadores es una materia aún no resuelta a cabalidad en Chile, lo cual no supone necesariamente la implementación de medidas de orden legislativo, en razón de las proyecciones sociales, culturales y económicas que supone el cambio de paradigma 
de "hombre proveedor y mujer cuidadora" hacia el anhelado modelo de "hombre y mujer proveedores y cuidadores". Esto última afirmación admite excepciones obvias, tratándose de materias específicas en las que el actual ordenamiento jurídico persiste en el arcaico pacto de producción y reproducción, según aparece de manifiesto, por ejemplo, en las actuales normas del CdT. sobre protección a la maternidad, las que marginan al padre para que pueda asumir un rol más activo en el cuidado de sus hijos e imponen una solitaria carga a la mujer en lo que respecta al cuidado de los niños y su eventual realización en el plano familiar.

Por otra parte, cabe llamar la atención sobre la necesidad de avanzar en la adecuación normativa de los principios y disposiciones del convenio № 156 de la OIT en nuestro país, materia en la cual se puede constatar un notable retraso y la ausencia de políticas claras y consistentes que partan de la base de la igualdad material de hombres y mujeres en todos los ámbitos de la vida social, como asimismo, que promuevan un cambio de roles y la sanción efectiva de las prácticas discriminatorias en materia laboral. Precisamente, en esta perspectiva, las modalidades atípicas de contratación laboral, como es el caso del teletrabajo, pueden cumplir una función renovadora e integradora que armonice los intereses de trabajadores, trabajadoras y empleadores, minimizando el impacto que la conciliación de trabajo y familia tiene para las empresas al generar, en el esquema normativo tradicional, una suspensión de la relación laboral (v. gr. permiso postnatal) o interrupciones en la jornada de trabajo (v. gr. derecho a dar alimentos). Asimismo, es evidente que las formas más flexibles de contratación laboral reducen el impacto negativo de la armonización de trabajo y familia para las mujeres, al no presentarse la maternidad como una decisión que las coloque en la disyuntiva de tener que postergar su realización profesional o familiar. De igual manera, las nuevas modalidades de contratación laboral como el teletrabajo se pueden convertir en un aliciente para que los hombres comiencen a asumir más activamente su paternidad, sin alejarse de sus actividades laborales habituales. Por consiguiente, se generan ventajas y oportunidades que pueden ayudar a revertir los actuales casos de discriminación laboral que afectan a mujeres y hombres en razón de la asunción de sus responsabilidades familiares, lo cual puede obrar naturalmente en provecho de los niños y niñas o de otras personas que requieran cuidado.

Finalmente, cabe afirmar que la compatibilización de la vida familiar y laboral no es una materia que involucre sólo a las mujeres trabajadoras para que los hombres asuman un rol más activo frente a las responsabilidades derivadas del cuidado de los hijos o de otros familiares directos, sino que, más bien, es un tema que incluye a toda la sociedad y, en importante medida, dice relación con el proyecto o modelo de sociedad que se pretende construir en 
el país. Desde esta perspectiva, un país como el nuestro que aspira a alcanzar el desarrollo y el consecuente bienestar económico y social de las naciones que se ubican en dicha escala, debe plantearse activamente temas como éste y transformarlos en un principio orientador del modelo a seguir, por su innegable vinculación con los valores de la igualdad de oportunidades, la solidaridad y la justicia social, sin los cuales el crecimiento económico se puede transformar en un concepto vacío y, por lo mismo, carente de significado para la mayoría de la población que debe enfrentar cada día los múltiples -y muchas veces injustos- desafíos de la actual vida en sociedad.

[Recibido el 27 de octubre y aprobado el 8 de noviembre de 2010].

\section{BiBLIOGRAFÍA}

Barrero Fernández, Antonio, El teletrabajo (Madrid, Editorial Ágata, 1999).

Bentancor, Andrea - CaAmaño Rojo, Eduardo, Protección a la maternidad: desafios y oportunidades, Columna de opinión publicada en el Diario La Segunda, miércoles 21 de julio de 2010.

CaAmaño Rojo, Eduardo, La actual regulación de la jornada de trabajo y su necesidad de flexibilización, en Revista de Derecho de la Pontificia Universidad Católica de Valparaíso, 28 (semestre I de 2007).

CaAmaño Rojo, Eduardo, Las transformaciones del trabajo, la crisis de la relación laboral normal y el desarrollo del empleo atípico, en Revista de Derecho de la Universidad Austral de Chile, 18 (julio 2005).

CAAmaño Rojo, Eduardo, Los efectos de la protección a la maternidad para la concreción de la igualdad de trato entre hombres y mujeres en el trabajo, en Revista de Derecho de la Pontificia Universidad Católica de Valparaíso, 33 (semestre II de 2009).

CAAMaño Rojo, Eduardo, Mujer y trabajo: origen y ocaso del modelo del padreproveedor y la madre cuidadora, en Revista de Derecho de la Pontificia Universidad Católica de Valparaíso, 34 (semestre I de 2010).

Comisión Asesora Presidencial "Mujer, Trabajo y Maternidad”, Informe final, documento disponible en www.sernam.cl.

Consejo Económico y Social, El trabajo a tiempo parcial, Informe No 4 (Sesión ordinaria del pleno de fecha 18 de septiembre de 1996, Madrid, 1996).

Dessler, Gary, Administración de personal, (8a edición, México, Editorial Pearson, 2001).

Díaz, Ximena - Todaro, Rosalba, Riesgos e inseguridades de las nuevas formas de uso flexible del tiempo de trabajo, en Serie En Foco, 35 (2004), disponible en: http://www. expansiva.cl/publicaciones/en_foco/detalle.tpl?iddocumento $=06102004132859$

Dirección del Trabajo, Encuesta Laboral 2008: Inequidades y brechas de género en el empleo, disponible en: http://www.dt.gob.cl/documentacion/1612/w3-article97629.html.

Gamonal Contreras, Sergio, Fundamentos de Derecho Laboral (Santiago, Editorial LexisNexis, 2008). 
Gamonal Contreras, Sergio, Trabajo y Derecho (Santiago, Editorial Legal Publishing, 2010).

Henrí euez, Helia - Cárdenas, Ana - Selamé, Teresita, Trabajo a domicilio en el siglo XXI. Tres miradas sobre el teletrabajo, en Cuaderno de Investigación, 26 (Santiago, Dirección del Trabajo, 2005).

Jáuregui Atondo, Ramón - Moltó García, Juan Ignacio - González de LenA, Francisco, Un futuro para el trabajo en la nueva sociedad laboral (Valencia, Editorial Tirant lo Blanch, 2004).

Plá Rodríguez, Américo, Los principios del Derecho del Trabajo ( $3^{\text {a }}$ edición, Buenos Aires, Editorial Depalma, 1998).

Raso Delgue, Juan, Las transformaciones del trabajo, documento disponible en: www. rau.edu.uy.universidad/instderechodeltrabajo/raso.pdf.

Riguelme, Verónica, El tiempo de trabajo, en Temas Laborales, 11, Dirección del Trabajo, documento disponible en: www.dt.gob.cl.

Salazar Concha, Cristian, El teletrabajo como un aporte a la inserción laboral de personas con discapacidad en Chile: una gran carretera virtual por recorrer, en Revista Ciencia \& Trabajo, 25 (julio-septiembre 2007), disponible en www.cienciaytrabajo. cl.

Thibault Aranda, Javier, El teletrabajo. Análisisjurídico laboral (2a edición, Madrid, Consejo Económico y Social, 2001).

Ugarte Cataldo, José Luis, Derecho del Trabajo, flexibilidad laboral y análisis económico del Derecho (Santiago, Editorial LexisNexis, 2004).

Ugarte Cataldo, José Luis, Elnuevo Derecho del Trabajo (Santiago, Editorial Lexis Nexis, 2007).

Walker Errázuriz, Francisco, Derecho de las relaciones laborales (Santiago, Editorial Universitaria, 2003). 